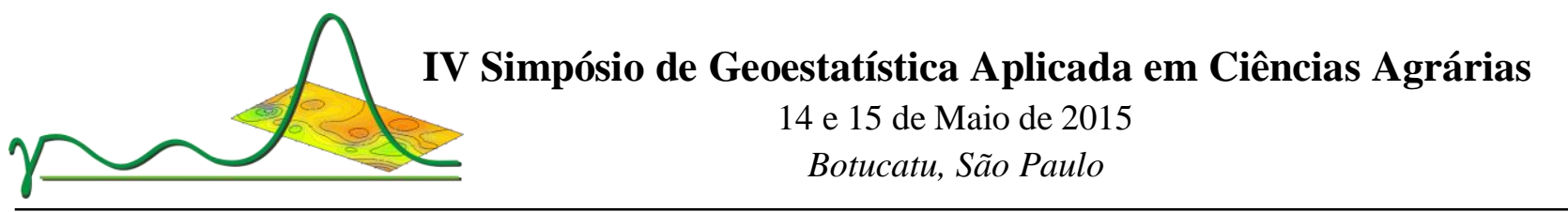

\title{
SIMULAÇÃO ESTOCÁSTICA NA DETERMINAÇÃO DO CENÁRIO CRÍTICO DA CONCENTRAÇÃO ESTIMADA DE TEBUTHIURON NA ÁGUA SUBTERRÂNEA
}

\author{
Moraes $^{1}$, D. A. C., Spadotto 2 , C.A., Zimback ${ }^{3}$, C. R. L. \\ ${ }^{1}$ Doutorando em Irrigação e Drenagem, Faculdade de Ciências Agronômicas/UNESP - Rua José Barbosa de Barros, \\ 1780 CEP: 18610-307 Botucatu, SP - Brasil. Caixa-Postal: 237 diegomoraes@ fca.unesp.br \\ ${ }^{2}$ Engenheiro Agrônomo, Ph.D., Embrapa Gestão Territorial - Av. Soldado Passarinho, 303, Fazenda Chapadão, \\ Campinas - SP, Brasil. claudio.spadotto@embrapa.br \\ ${ }^{3}$ Profa. Adjunta do Departamento de Recursos Naturais, FCA/UNESP - Rua José Barbosa de Barros, 1780 CEP: \\ 18610-307 Botucatu, SP - Brasil. Caixa-Postal: 237, czimback@gmail.com
}

\begin{abstract}
Resumo - O presente trabalho teve como objetivo avaliar a aplicação da Simulação Gaussiana Sequencial (SGS) para determinar o cenário crítico da concentração estimada do Tebuthiuron na água subterrânea e compará-la com o padrão de potabilidade. Para tanto, foi considerada uma malha irregular, em uma bacia hidrográfica, composta de 100 amostras com atributos climáticos, do solo (profundidade de $0-20 \mathrm{~cm}$ e $20-40 \mathrm{~cm}$ ) e do corpo de água subterrâneo. Também foram consideradas as propriedades do Tebuthiuron. Os valores das amostras foram inseridos no software ARAquá para a obtenção das concentrações estimadas do Tebuthiuron na água subterrânea. Com isso, foi realizada a análise estatística e variográfica, validação cruzada e os procedimentos da SGS. O número de realizações na SGS foi de 100. O cenário crítico obtido pela SGS mostrou que a maior concentração estimada do Tebuthiuron na água subterrânea foi de 3,36 ug. $\mathrm{L}^{-1}$, não excedendo o valor para o padrão de potabilidade em toda área de estudo.
\end{abstract}

Palavras-chave: Defensivos Agrícolas; Recursos Hídricos; Simulação Gaussiana Sequencial.

\section{STOCHASTIC SIMULATION ON DETERMINING CRITICAL SCENARIO FOR TEBUTHIURON ESTIMATED CONCENTRATION IN GROUNDWATER}

\begin{abstract}
This work aims to assess the Sequential Gaussian Simulation (SGS) application to determine the critical scenario for Tebuthiuron estimated concentration in groundwater and compare with drinking water standard. It was considered a irregular mesh, in a watershed, composed by 100 weather, soil $(0-20 \mathrm{~cm}$ and $20-40$ depth $)$ and groundwater attributes. Also, pesticide properties were considered. The attribute values were inserted into the ARAquá software for obtaining estimated concentrations of Tebuthiuron in groundwater. After, statistical and geostatistical analysis, cross validation and SGS procedures were performed. The realization number in SGS were set at 100. The critical scenario obtained by SGS showed that the highest Tebuthiuron estimated concentration in groundwater was 3,36 ug. $\mathrm{L}^{-1}$, which not exceed the drinking water standard for entire study area.
\end{abstract}

Key words: Pesticides; Water Resources; Sequential Gaussian Simulation.

\section{Introdução}

Sabe-se que o uso indevido de defensivos agrícolas pode causar danos ao ambiente, bem como aos organismos que nele se encontram. Os herbicidas são apontados como o grupo de defensivos agrícolas mais frequentemente detectados em estudos de qualidade de águas superficiais e subterrâneas (CARTER, 2000).

Para auxiliar na tomada de decisão no registro e ordenamento do uso de defensivos agrícolas são utilizados modelos matemáticos, os quais têm sido empregados com o objetivo de prever o comportamento e o destino ambiental desses produtos químicos. Em conjunto com esses modelos matemáticos, técnicas de espacialização presentes em Sistemas de Informações Geográficas (SIG) e geoestatística têm sido empregadas em estudos do comportamento ambiental de defensivos agrícolas. Nicolella (1999) utilizou o software CMLS e krigagem ordinária na avaliação do risco de contaminação do lençol freático por Tebuthiuron. Moraes, Zimback e Spadotto (2012) obtiveram a variabilidade espacial das estimativas de concentração do Tebuthiuron na água subterrânea utilizando o software ARAquá e o método de interpolação por krigagem ordinária.

Observa-se que os trabalhos citados utilizaram a krigagem ordinária como procedimento de interpolação dos resultados obtidos pelos modelos matemáticos. No entanto, um problema relacionado ao esse processo é o efeito de suavização das espacializações, o qual não reproduz adequadamente as características da amostra utilizada para realizar 


\section{Simpósio de Geoestatística Aplicada em Ciências Agrárias \\ 14 e 15 de Maio de 2015 \\ Botucatu, São Paulo}

as estimativas em pontos não amostrados, sendo a simulação estocástica a solução adotada para resolver o impasse da suavização (YAMAMOTO; LANDIM, 2013).

Nesse contexto, este trabalho foi realizado com o objetivo de avaliar a aplicação da Simulação Gaussiana Sequencial (SGS) nos resultados, para determinar o cenário crítico da concentração estimada do Tebuthiuron na água subterrânea e compará-lo com o padrão de potabilidade.

\section{Material e Métodos}

Localizada na região centro sul do Estado de São Paulo, a Bacia do Rio Pardo abrange áreas dos municípios de Botucatu e Pardinho. A precipitação e evapotranspiração anual são de 1.399 mm (CIIAGRO, 2014) e 884 mm (AGRITEMPO, 2014), respectivamente.

Foram utilizadas 100 amostras de solo, baseadas no levantamento realizado por Zimback (1997). Os parâmetros considerados como dados de entrada no software ARAquá, para o solo, foram: capacidade de campo (volume.volume ${ }^{-}$ ${ }^{1}$ ), carbono orgânico (massa.massa $\left.{ }^{-1}\right)$, densidade $\left(\mathrm{g} . \mathrm{cm}^{-3}\right)$ e porosidade na zona saturada (volume.volume $\left.{ }^{-1}\right)$.

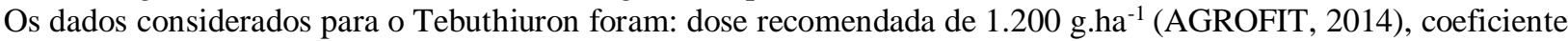
de sorção normalizado para a fração de carbono orgânico $\left(\mathrm{K}_{\mathrm{oc}}\right)$ de $80 \mathrm{~mL} \cdot \mathrm{g}^{-1}$ e meia vida de 360 dias (SPADOTTO; GOMES; HORNSBY, 2002). O padrão de potabilidade adotado é de 500 ug.L-1 (EXTOXNET, 1993).

As amostras foram submetidas aos cálculos do software ARAquá (SPADOTTO, 2010), resultando, em cada ponto amostral, na concentração estimada do Tebuthiuron na água subterrânea.

Após a obtenção das concentrações estimadas na água subterrânea pontualmente, as mesmas foram submetidas à estatística descritiva e ao procedimento da SGS no software ISATIS 2013 (GEOVARIANCES, 2013). No entanto, a SGS requer que os dados possuam distribuição normal. Para tanto, as concentrações estimadas foram submetidas à transformação para distribuição normal por meio da anamorfose gaussiana (WACKNARGEL, 2003). Com isso foi estimado o variograma com base na pressuposição de estacionaridade da hipótese intrínseca, a qual é estimada pela Equação 1, segundo Journel (1989):

$$
\gamma(h)=\left(\frac{1}{2 N(h)}\right) \sum_{i=1}^{N(h)}\left[z\left(x_{i}\right)-z\left(x_{i}+h\right)\right]^{2}
$$

onde, $\mathrm{N}(\mathrm{h})$ é o número de pares experimentais de observações e $\mathrm{Z}(\mathrm{xi})$ e $\mathrm{Z}(\mathrm{xi}+\mathrm{h})$ são os pares de pontos separados por uma distância $h$.

Foi analisado o grau de dependência espacial utilizando o Índice de Dependência Espacial (IDE), proposto por Zimback (2001), onde valores de IDE $\leq 25 \%$ indicam fraca dependência espacial; $25 \%<$ IDE $<75 \%$ moderada dependência espacial e IDE $\geq 75 \%$ forte dependência espacial. Após o ajuste, o variograma foi submetido ao processo de validação cruzada com o objetivo de verificar se a modelagem do variograma é satisfatória e a krigagem possui caráter não enviesado e mínima variância de estimação. Para tanto, foram considerados o coeficiente de correlação entre os valores observados e estimados, erro médio (EM) (Equação 2), e a variância do erro padronizado (VEP) (Equação 3):

$$
E M=\frac{1}{N} \sum\left(z\left(x_{i}\right)-z^{*}\left(x_{i}\right)\right)
$$

onde, $N$ é o número de amostras; $z\left(x_{i}\right)$ é o valor observado; $z^{*}\left(x_{i}\right)$ é o valor estimado. $\mathrm{O}$ erro médio demonstra o grau de não-enviesamento e seu valor deve ser próximo de zero (CASTRIGNANÒ, 2011).

$$
V E P=\frac{1}{N} \sum\left(\frac{\left(z\left(x_{i}\right)-z^{*}\left(x_{i}\right)\right.}{\sigma_{i}}\right)^{2}
$$

onde, $N$ é o número de amostras; $z\left(x_{i}\right)$ é o valor observado; $z^{*}\left(x_{i}\right)$ é o valor estimado. A variância do erro padronizado demonstra a razão entre a variância experimental e teórica, sendo que seu valor deve ser próximo de 1 (CASTRIGNANÒ, 2011), dentro de um intervalo de tolerância (IT), conforme proposto por Chilès e Delfine (1999) na Equação 4:

$$
I T=1 \pm 3 \sqrt{\frac{2}{N}}
$$

onde, $N$ é o número de amostras.

Com isso, foi realizado o procedimento da SGS conforme os passos definidos por Gebbers e Bruin (2010) e resumidos na Figura 1. 


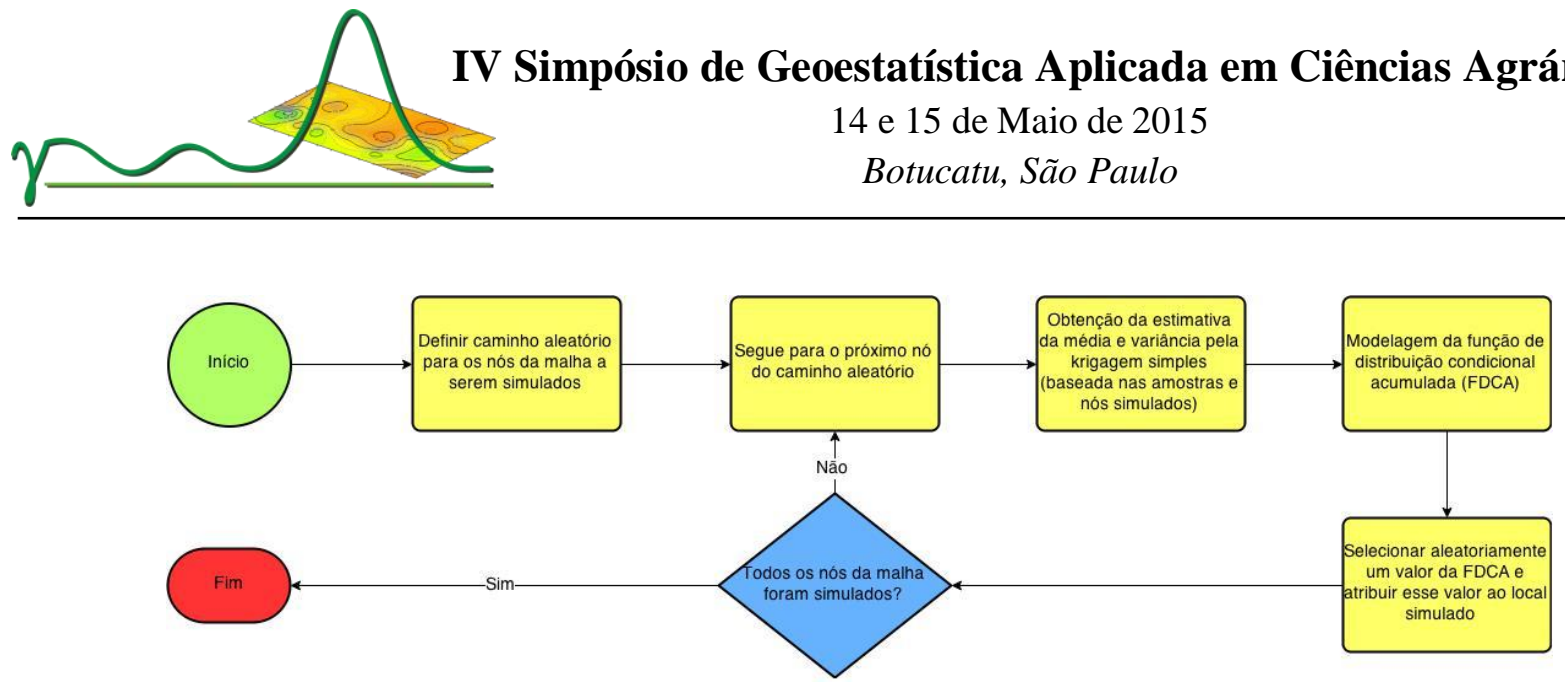

Figura 1. Fluxograma dos processos executados pela SGS.

Foi necessária a escolha do número de realizações da SGS, sendo aplicadas nesse estudo 2, 50, 100 e 500 realizações. A escolha do número de realizações foi baseada na estabilização dos valores da variância entre as espacializações obtidas.

O pós-processamento foi aplicado às realizações de forma a obter o maior valor para a concentração estimada do Tebuthiuron na água subterrânea.

\section{Resultados e Discussão}

A estatística descritiva para a concentração estimada do Tebuthiuron na água subterrânea é apresentada na Tabela 1.

Tabela 1. Estatística descritiva da concentração estimada do Tebuthiuron na água subterrânea.

\begin{tabular}{|c|c|c|c|c|c|c|}
\hline Parâmetro & $\begin{array}{l}\text { Mínimo } \\
\left(\text { ug.L }{ }^{-1}\right)\end{array}$ & $\begin{array}{c}\text { Máximo } \\
\left.\text { (ug.L }{ }^{-1}\right)\end{array}$ & $\begin{array}{c}\text { Média } \\
\left(\text { ug.L }{ }^{-1}\right)\end{array}$ & Desvio-Padrão & Variância & $\begin{array}{c}\text { Coeficiente } \\
\text { de Assimetria }\end{array}$ \\
\hline CEDNT $^{*}$ & 0,00 & 3,36 & 0,25 & 0,57 & 0,33 & 3,75 \\
\hline CEDT $^{* *}$ & $-2,41$ & 2,41 & 0,00 & 0,99 & 0,97 & 0,00 \\
\hline
\end{tabular}

É possível notar que o valor máximo obtido para a concentração estimada do Tebuthiuron na água subterrânea não excedeu o valor máximo para o padrão de potabilidade $\left(500 \mathrm{ug} . \mathrm{L}^{-1}\right)$. Para os dados transformados, nota-se que a anamorfose gaussiana foi eficaz, uma vez que a média possui valor igual a 0 , a variância próxima a 1 e o coeficiente de assimetria possui valor igual a 0 (distribuição simétrica).

O variograma da concentração estimada do Tebuthiuron na água subterrânea é apresentado na Figura 2.

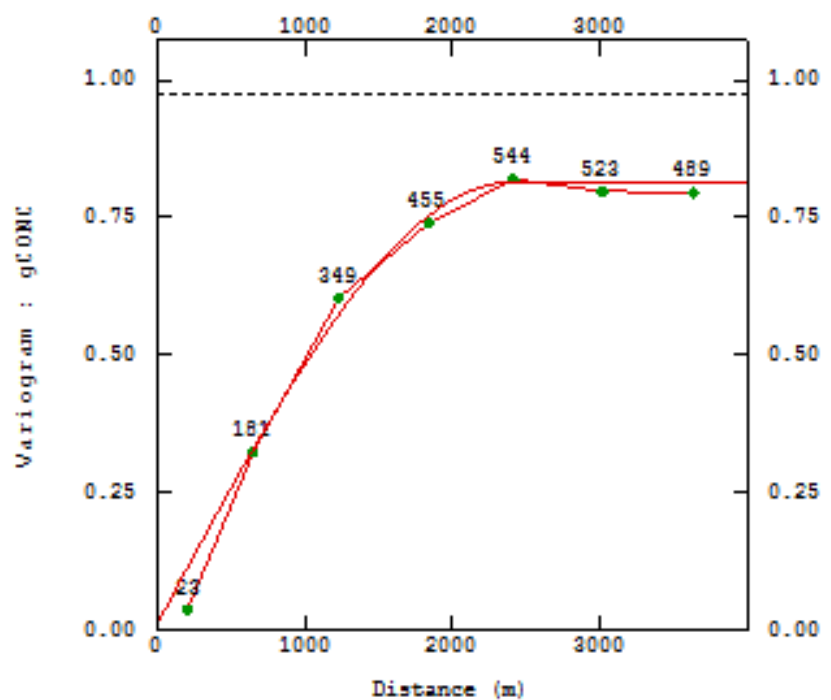

Figura 2. Variograma da concentração estimada do Tebuthiuron na água subterrânea.

O variograma apresentou ajuste ao modelo experimental com o modelo esférico, com um alcance de 2.400 metros. O IDE obteve o valor de $98,75 \%$, indicando forte dependência espacial. 


\section{Simpósio de Geoestatística Aplicada em Ciências Agrárias \\ 14 e 15 de Maio de 2015 \\ Botucatu, São Paulo}

Os resultados da validação cruzada para concentração estimada do Tebuthiuron na água subterrânea são apresentados na Tabela 2.

Tabela 2. Validação cruzada para concentração estimada do Tebuthiuron na água subterrânea.

\begin{tabular}{cccc}
\hline Coeficiente de Correlação & EM & VEP & IT \\
\hline 0,880 & $-0,009$ & 0,823 & $0,575-1,424$ \\
\hline
\end{tabular}

Os valores do erro médio foram próximos de 0 , indicando um baixo grau de enviesamento. Já a variância do erro padronizado encontra-se próxima de 1 e dentro do intervalo de tolerância. Os valores de correlação indicam que os valores estimados pelo modelo do variograma foram próximos aos valores reais. Portanto, o ajuste do modelo do variograma é aceitável para realizar a SGS.

Em relação ao número de realizações na SGS, o gráfico apresentado na Figura 3a mostra que o ponto de estabilização dos valores da variância entre os mapas foi em 50 realizações. No entanto, nota-se que ocorreu uma pequena flutuação na variância após 50 realizações, voltando a estabilizar em 100 realizações. Tal fato levou à escolha de 100 realizações como procedimento de segurança, uma vez que após 100 realizações a variância estabiliza totalmente.

A espacialização do cenário crítico para a concentração estimada do Tebuthiuron na água subterrânea é apresentada na Figura 3b.

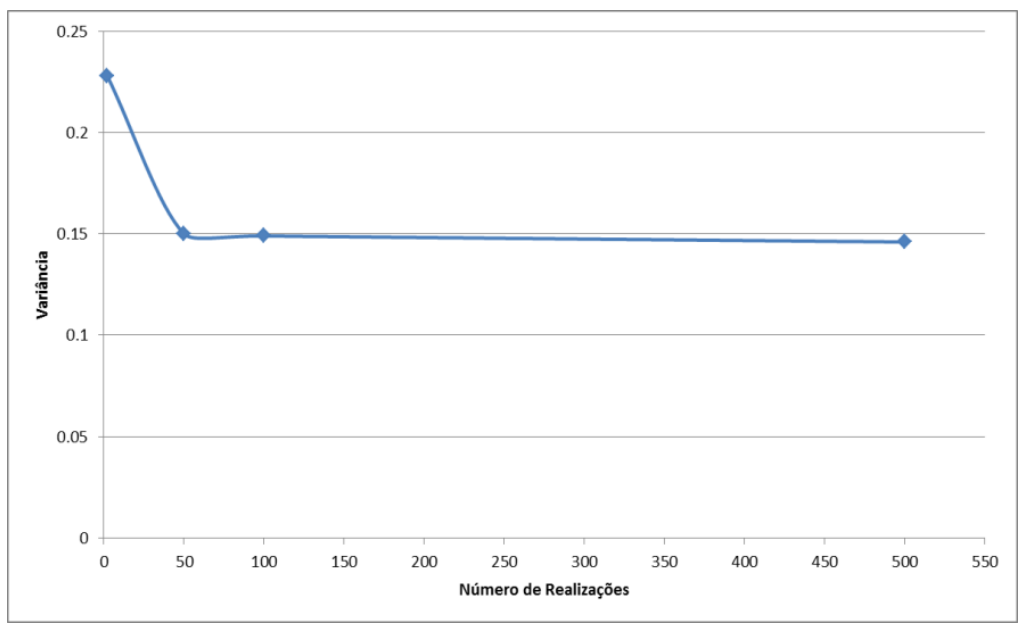

(a)

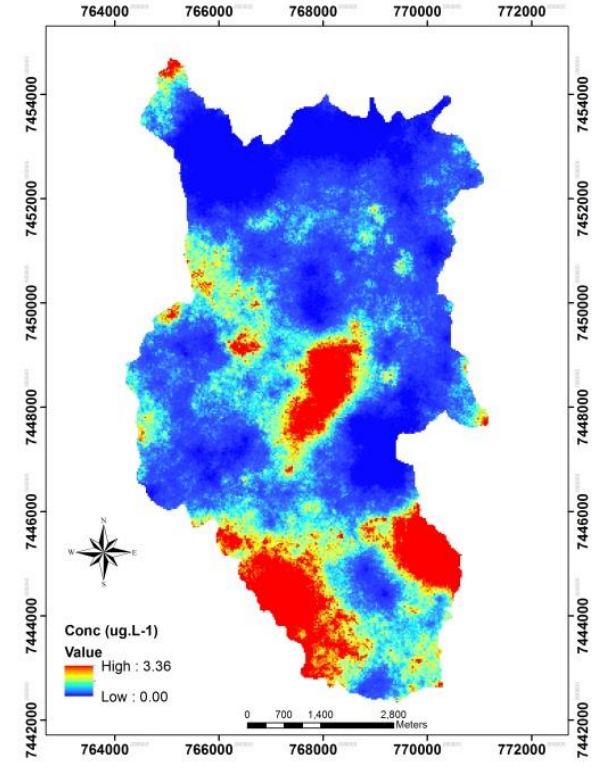

(b)

Figura 3. Gráfico da relação entre o número de realizações na SGS e a variância entre as mesmas (a) e espacialização das concentrações estimadas do Tebuthiuron na água subterrânea no cenário crítico (b).

Conforme explicitado por Yamamoto e Landim (2013), não há suavização da espacialização. Dessa forma, a estrutura de correlação espacial foi preservada, resultando em um mapa mais realístico. Porém, afirmar que as espacializações obtidas por SGS são próximas da realidade não significa afirmar que são acuradas, uma vez que Castrignanò et al. (2002) e Teixeira et al. (2011) concluíram que as espacializações oriundas da krigagem são mais acuradas em relação à SGS.

Assim como na estatística descritiva, a espacialização demonstrou que a menor concentração obtida no cenário crítico foi nula e a maior concentração foi de 3,36 ug. $\mathrm{L}^{-1}$. Tal situação não é observada quando se utiliza a krigagem, uma vez que esta superestima valores baixos e subestima valores altos, situação que pode não caracterizar um cenário crítico na avaliação de risco ambiental de defensivos agrícolas.

\section{Conclusões}

Em face dos resultados obtidos neste estudo, pode-se concluir que:

- O uso da SGS permitiu a obtenção do cenário mais crítico para a concentração estimada do Tebuthiuron na água subterrânea, sendo 100 o número de realizações empregadas no procedimento. 


\section{Simpósio de Geoestatística Aplicada em Ciências Agrárias \\ 14 e 15 de Maio de 2015 \\ Botucatu, São Paulo}

de potabilidade.

- Mesmo em cenário crítico a concentração estimada de Tebuthiuron na água subterrânea não excedeu o padrão

- A SGS aplicada na avaliação de risco ambiental de defensivos agrícolas auxilia a tomada de decisão, uma vez que preserva a variabilidade espacial do parâmetro em estudo e mantém seu histograma amostral.

- Futuros trabalhos devem considerar outros defensivos agrícolas e a variabilidade espacial da precipitação, evapotranspiração e irrigação anual.

\section{Agradecimentos}

Ao Grupo de Estudos e Pesquisas Agrárias Georreferenciadas (GEPAG), Coordenadoria de Aperfeiçoamento de Pessoal de Nível Superior (CAPES - Processo 99999.002573/2014-08 PDSE) pelo apoio financeiro, à Embrapa Gestão Territorial pelo apoio técnico-científico, e ao Consiglio per la Ricerca e la Sperimentazione in Agricoltura - CRA SCA pela utilização do software ISATIS.

\section{Referências}

AGRITEMPO. Sistema de monitoramento agrometeorológico. 2014. Disponível em: <http://www.agritempo.gov.br/agritempo/index.jsp>. Acesso em: 01 ago. 2014.

AGROFIT. Sistema de Agrotóxicos Fitossanitários. Consulta de Ingrediente Ativo. 2003. Disponível em: <http://extranet.agricultura.gov.br/agrofit_cons/principal_agrofit_cons>. Acesso em: 01 ago. 2014.

CARTER, A. D. Herbicide movement in soils: principles, pathways and processes. Weed Res., S.i., v. 40, p. 113-122, 2000.

CASTRIGNANÒ A. et al. Estimation or stochastic simulation in soil science. In : ZDRULI P., STEDUTO P., KAPUR S.. 7. International meeting on Soils with Mediterranean Type of Climate. Bari : CIHEAM, 2002 -p. 167 -1 82.

CASTRIGNANÒ, A.. Introduction to spatial data processing. 1. ed. Roma: Aracne, 2011. 108 p.

CENTRO INTEGRADO DE INFORMAÇÕES AGROMETEOROLÓGICAS. Monitoramento agrometeorológico e climático. 2014. Disponível em: <http://www.ciiagro.sp.gov.br/>. Acesso em: 01 ago. 2014.

CHILÈS, J.P., DELFINER, P.. Geostatistics: Modeling Spatial Uncertainty. New York: John Wiley \& Sons, Inc., 1999. 721p.

EXTENSION TOXICOLOGY NETWORK. Tebuthiuron. 1993. Disponível em:

<http://pmep.cce.cornell.edu/profiles/extoxnet/pyrethrins-ziram/tebuthiuron-ext.html〉. Acesso em: 19 dez. 2014.

GEBBERS, R.; BRUIN, S.. Application of Geostatistical Simulation in Precision Agriculture. In: OLIVER, M. A.. Geostatistical Applications for Precision Agriculture. London: Springer, 2010. p. 269-301.

GEOVARIANCES. ISATIS Technical Ref., ver. 2013.1. Geovariances \& Ecole des Mines de Paris. Avon Cedex. Paris. 2013.

JOURNEL, A. G..Fundamentals of geostatistics in five lessons. 1989. Washington: American geophysical union. 1989. 134p.

MORAES, D. A. C.; ZIMBACK, C. R. L.; SPADOTTO, C. A.. Espacialização das estimativas de contaminação de águas subterrâneas por Tebuthiuron. Irriga, Botucatu, v. 17, n. 3, p.299-312, set. 2012.

NICOLELLA, G.. Avaliação de risco de contaminação do lençol freático, pelo herbicida Tebuthiuron em cana-deaçúcar, utilizando geoestatística e simulador CMLS.1999. 151 f. Tese (Doutorado) - Curso de Engenharia Agrícola, Universidade Estadual de Campinas, Campinas, 1999.

SPADOTTO, C. A.; GOMES, M. A. F.; HORNSBY, A.. Pesticide Leaching Potential Assessment in Multi-layered Soils. 2002. Disponível em: <http://ojs.c3sl.ufpr.br/ojs/index.php/pesticidas/article/view/3145/2518>. Acesso em: 19 dez. 2014. 


\section{Simpósio de Geoestatística Aplicada em Ciências Agrárias \\ 14 e 15 de Maio de 2015 \\ Botucatu, São Paulo}

SPADOTTO, C. A. et al. ARAquá: programa para avaliação de risco ambiental de agrotóxico. Campinas: Embrapa Monitoramento por Satélite, 2010. 15 p.

TEIXEIRA, D. B. et al. Avaliação da krigagem ordinária e simulação sequencial gaussiana na interpolação da emissão de CO2 do solo. In: II SIMPÓSIO DE GEOESTATÍSTICA APLICADA EM CIÊNCIAS AGRÁRIAS, 3., 2011, Botucatu. Anais... . Botucatu: Fepaf, 2011. p. 1 - 6.

WACKERNAGEL, H.. Gaussian Anamorphosis with Hermite Polynomials. In: WACKERNAGEL, H.. Multivariate Geostatistics: An Introduction with Applications. Berlin: Springer, 2003. p. 238-249.

YAMAMOTO, J. K.; LANDIM, P. M. B.. Geoestatística: Conceitos e Aplicações. São Paulo: Oficina de Textos, 2013. $215 \mathrm{p}$.

ZIMBACK, C. R. L. Levantamento semidetalhado dos solos da bacia do Rio Pardo nos Municípios de Pardinho e Botucatu. Botucatu: FEPAF, FCA-UNESP, 1997. 55 p.

ZIMBACK, C. R. L. Análise espacial de atributos químicos de solos para fins de mapeamento da fertilidade. 2001. 114 f. Tese (Livre-Docência em Levantamento do solo e Fotopedologia)-Faculdade de Ciências Agronômicas, Universidade Estadual Paulista, Botucatu, 2001. 